\title{
Review of Closed Circuit Television (CCTV) Techniques for Vehicles Traffic Management
}

\author{
Heba A. Kurdi \\ Al Imam Muhammad Ibn Saud Islamic University \\ Riyadh, Saudi Arabia
}

\begin{abstract}
Due to the population increase all over the world current road infrastructures are unable to keep up with the escalating transportation demands. This situation where travel demands exceeds the capacity of the transportation network is known as traffic congestion. However, it is impractical to build more roads and infrastructure to accommodate these demands. Governments are increasingly recognizing the importance of traffic control tools, such as Closed Circuit Television (CCTV) systems as a feasible solution to mitigate the traffic congestion problem. CCTV systems are deployed across city centres, motorways, trunk roads, car parks etc., to collect diverse data on large regions where manual observation can be difficult, problematic or unfeasible. By processing this data, which contains video images of traffic parameters, useful information can be extracted, including speed, traffic composition, vehicle shapes, vehicle types, vehicle identification numbers and occurrences of traffic violations or road accidents.
\end{abstract}

This paper reviews different approaches to utilize CCTV systems for traffic management of Vehicles. It highlights existing architectural, deployment models, and various approaches to analyse generated traffic data. The objective is to provide a clear background that can help in any related future research.

\section{KEYWORDS}

CCTV, Traffic control, sensors network, embedded systems.

\section{INTRODUCTION}

Closed circuit television (CCTV) refers to the use of video cameras to transmit signals to a specific place with a set of monitors, as shown in Figure 1. Nowadays CCTV plays a significant role in protecting the public and implementing security. It is increasingly used by many countries for critical applications, such as bank monitoring, retail control and crime detection, where manual monitoring can be difficult, problematic or unfeasible [1][2][3]. For instance, the number of CCTV cameras in the United Kingdom is estimated in the range from three to six million, dispersed across motorways, trunk roads, car parks, shops, hospitals, airports, train stations, streets etc [4]. 


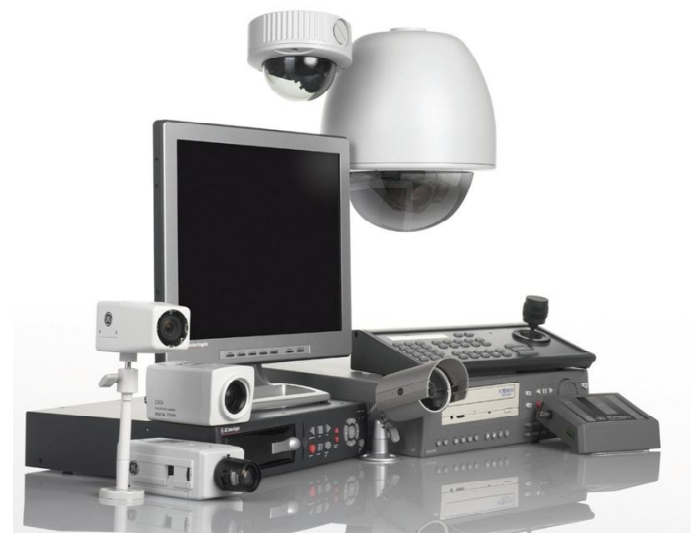

Figure 1: Main parts of CCTV

As world population increases, traffic control is becoming a critical application for CCTV. CCTV provides a way to monitor multiple cameras internally and analyse generated images to extract useful information [5] about traffic parameters, such as speed, traffic composition, vehicle shapes, vehicle types, vehicle identification numbers and occurrences of traffic violations or road accidents. This offers a great help for transportation authorities, allowing them to make decisions accordingly and distribute traffic information to drivers [6], resulting in improved traffic flow, prompt accident detection, shorter journey time, less fuel consumption, reduced emissions and more satisfied travelers [7][8].

This paper presents a review on vehicles traffic management by the CCTV technology. It highlights existing architectural and deployment models, as well as the various approaches utilised to analyse generated traffic data. However, due to the great variety in CCTV deployment models and analysis approaches, this literature review by no means intends to be a comprehensive study. Instead, the objective is to shed some light on these models and approaches to promote future research in this area.

In the context of vehicles traffic management, there are two different perspectives to be considered: the driver's perspective and the traffic authority's perspective. As far as drivers are concerned, the quickest and shortest path is usually preferred. This paper focuses on the perspective of the traffic authority, which is more interested in road safety and eliminating congestion. Additionally, although traffic control applies equally to vehicles and pedestrians, this paper considers pedestrians traffic control as out-of-scope, due to time constraints.

The rest of this paper is structured as follows: Section 2 illustrates the structural and architectural elements of CCTV systems for traffic control applications. Deployments models and issues are discussed in Section 3. Data analysis steps and reaction approaches are presented in Section 4 and Section 5, respectively. Section 6 touches on some considerations and legal issues associated with CCTV. Finally, Section 7 provides a brief summary and possible future research directions.

\section{SYSTEM ARCHITECTURE}

A CCTV system is a closed video system where the signal is transmitted to a limited set of monitors, restricting the view to a certain set of people with specific purposes. This is in contrast to broadcast TVs, where the signal is openly transmitted to unclosed recipients. 
CCTV systems vary greatly in size, starting from small systems installed in houses for private purposes and ending with extra-large systems spanning multiple continents [1]. A typical CCTV system, shown in Figure 2, is composed of the following:

- $\quad$ Camera System: all CCTV systems contain a group of cameras to monitor specific areas and capture images from them. Cameras used can be analogue or digital. However, analogue cameras suffer from low resolution, storage limitations and difficulty in searching through captured images. There are two types of CCTV cameras, based on the recording method: nonautomatic recording and automatic recording; the difference is that in the non-automatic recording cameras, there are no smart detectors to identify whether there is something worth recording or not, which requires a trained observer to monitor the videos. On the other hand, the automatic recording CCTV cameras include detectors to sense abnormal activities and consequently record them [9]. This has the great advantage of saving power as well as storage.

- $\quad$ Reviewing Aid: captured images can be viewed in real-time or recorded for later review [10]. This includes devices such as VHS video tapes, CDs, DVDs, tapes or a computer-based medium connected to a viewing monitor. Observers, on-site or off-site, can review captured images directly on screens or through telephone lines, the Internet or any other network [11].

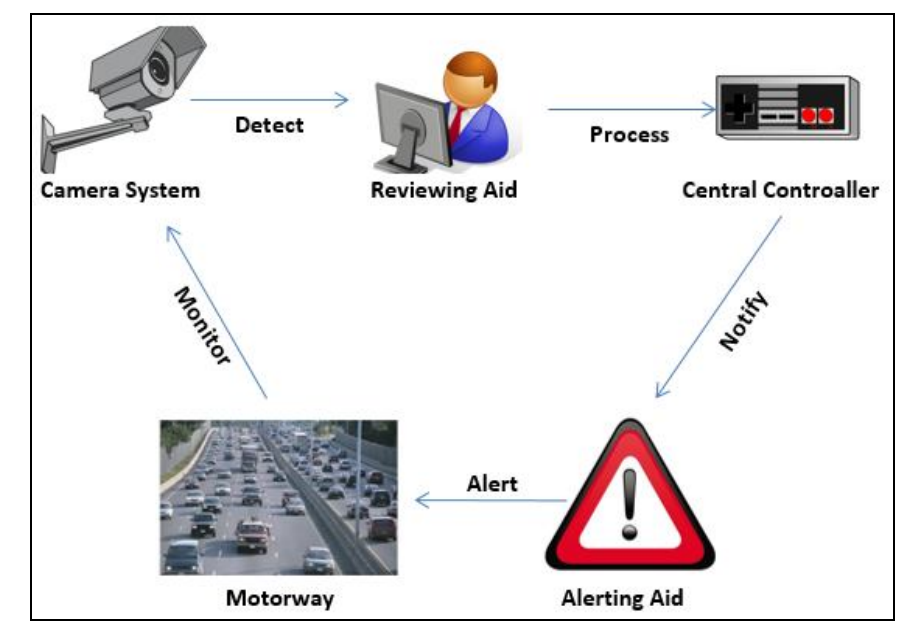

Figure 2: Main components and workflow in a traffic control CCTV system

- Central Controller: this part is responsible for pre-processing, processing and postprocessing of captured images. These functions can be carried out manually or automatically with the use of computer hardware and software tools, as will be described in the following section.

- $\quad$ Accessories: some modern CCTV systems come with accessories, such as extra lighting for recording in dark or difficult areas, and video-motion automatic detectors, which can be programmed to detect motion in a specific direction or objects of a specific size or speed [12].

\section{DEPLOYMENT MODELS}

For a CCTV system to estimate the traffic load dynamically and control it in real-time, it is essential to choose a suitable deployment model, which determines the number of cameras and their placement over road junctions. Three deployment models have been addressed in [13]: 
- $\quad$ Full installation: the CCTV cameras are installed at every junction. Although this model is the most expensive model, it proves to be the most effective one.

- $\quad$ Partial installation: only some junctions have cameras installed, based on certain criteria. For example, junctions known for their high frequency of accidents can have cameras. Other junctions will have no cameras and hence no traffic information.

- $\quad$ Optimum installation: only some junctions have cameras installed based on calculating the optimum number of cameras that best controls the entire network traffic. Although other junctions have no cameras, they can get traffic information from the junctions that have them. This deployment model is cost-effective even though it has partial installation. It has almost the same efficiency as that of the full installation.

However, for the best performance, full installation is preferable. To overcome the difficulty associated with constructing a new CCTV system by installing a large number of CCTV cameras to cover all road junctions, it is recommended to combine between the new CCTV system and an existing one. One of the studies that followed this approach is presented in [9]. It took place in the City of Leeds in the United Kingdom. It added new cameras to an existing CCTV system, which was already running by the city council. Based on this study, choosing an existing system has some disadvantages, because the system was not especially designed for the end objective. As a result, some existing cameras needed to be replaced and others had to be redirected to avoid obstructions.

Another issue that faces reusing existing CCTV systems is the possibility that the actual coverage provided by existing cameras does not equal the desired coverage. This means that some cameras will not be useful for the new system due to their locations. Therefore, their captured images should be excluded [13].

In general, making a decision whether and where to deploy a CCTV system is critical. All role players, including the police, emergency services and the local community should make an input in this decision, taking several factors into consideration. The main factors that should be considered when deploying a CCTV project include:

- Location of the project: whether it is situated in an urban area, large city or small town. Additionally, the fields of sight and availability of structures and places to install cameras.

- $\quad$ Available equipment and technology: There are numerous CCTV equipment in the market. Therefore, careful consideration should be taken when selecting them based on the context. For instance, whether the system needs to function in poor light will influence choice of equipment and technology.

- $\quad$ Characteristics of the traffic: By studying reoccurring traffic patterns and trends, it is possible to learn how they are formed and why. Some CCTV cameras can be more effective for certain traffic types. Additionally, certain traffic patterns might require special cameras or controlling mechanisms [14].

- Geography of the area: the physical characteristics and layout of streets. Some obstructions that can affect CCTV cameras deployment decisions include:

- $\quad$ Physical obstruction of buildings: This problem is clearer when roads are curved.

- Vegetation obstruction: such as that by long trees, so their removals might be necessary.

- $\quad$ Street accessories: for example, lamps and traffic lights and road signs. These are not a problem from a distance, but they are when zooming.

- Street alignment: The higher area of the street cannot be monitored if cameras are not high enough. 
- $\quad$ Large vehicles: may cause a temporary vision obstruction.

- $\quad$ Constructional area: usually have large winches and derricks that obscure vision [13].

\section{DATA ANALYSIS APPROACHES}

Initially, CCTV systems were managed manually, having people working as observers to analyse captured data and react accordingly. This process was error-prone and expensive in terms of time and cost. The use of digital cameras in CCTV systems has widened the utilisation of computer systems in analysing captured data and processing it. Two main approaches can be identified for computer analysis of CCTV in traffic control applications: sound-based systems and image-based systems.

\subsection{Sound-based CCTV Systems}

A Sound-based CCTV system contains one or more cameras with a directional microphone, a central controller and a video recorder. The sound is recorded constantly accompanied by the video on a memory unit. When the system hears an unusual sound, for instance a "crash-like" sound then, automatically, the pre- and post-accident recorded scenes are sent from the memory to the controller. The controller analyses the situation and identifies possible causes. This system was developed by Mitsubishi Electric Company in 1995, it was called the Traffic Accident Auto Memory System (TAAMS) or the Auto Incident Recording System (AIRS). Its first use was in Japan and then it was widely adopted in the USA [5] However, it seems like the amount of work available in this approach is still lacking when compared to image-based CCTV systems.

\subsection{Image-based CCTV Systems}

Image-based CCTV systems have the ability to recognise unusual and abnormal events on roads by analysing digital images and extracting traffic parameters such as speed and traffic composition. Special software tools are usually used to help in recognising vehicle shapes, vehicle types, vehicle identification numbers and occurrence of traffic violations or road accidents. Figure 3 shows the Automatic Number Plate Recognition (ANPR) system as an example of such tools.

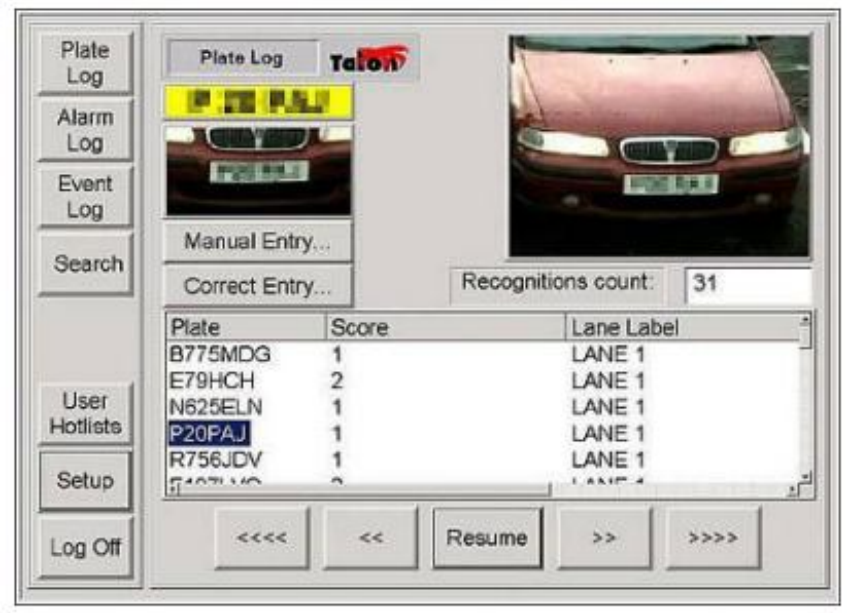

Figure 3. Software tool ANPR for automatic extraction of vehicle information 
There are four steps that are usually carried out by an image-based traffic control CCTV system to automatically analyse captured images:

- Pre-processing: in this step, irrelevant details and noises, such as shadows, light effects, rain and camera-moving effects, are reduced form captured images. This step is not mandatory, but is offered by some modern CCTV systems to provide results that are more accurate.

- $\quad$ Processing: the raw or pre-processed captured images are processed by searching for specific patterns, extracting relevant features and highlighting them. In [15], a framework for processing captured CCTV images from traffic control scenes was developed. It operates by detecting vehicles, based on either a motion mask or an image texture. The detector uses background estimation to model the static part of the scene after removing unnecessary details such as shadows

- $\quad$ Post-processing: having CCTV cameras continuously recording certain scenes will make reviewing them a difficult and time consuming task, because of their large amount. Therefore, some software tools have been suggested, such as BriefCam [4], which can automatically summarise the recorded scenes. The basic idea behind the software is that most CCTV cameras are stationary and so they record static backgrounds. As a result, when a moving object enters the scene, it can be detected and sections of the video including it are identified. Then, all events occurring in a given time period are superimposed in a short video that shows all of the actions at once. An operator or a reviewer needs only to view the summary video. If any unusual thing is spotted, he can click on it to jump straight to the relevant point in the original video.

\section{REACTION APPROACHES}

After processing captured data and detecting an incident, two different approaches are available in the literature to implement the system reaction:

- Manual reaction: where the workflow of the CCTV system does not include a specific component that directly communicates with road users or traffic authorities to notify them of hazards or traffic conditions. Rather, skilled observers frequently analyse captured scenes and make decisions accordingly. If actions were required, such as alerting road users, this would be carried out manually.

- $\quad$ Automatic reaction: some new CCTV computer analysis techniques include intelligent modules to react or make a decision in case of hazards. The CCTV system, beside its monitoring and detection tasks, also preforms the observer's job of notifying road users. This means that the CCTV system in this case has access to smart road signs or traffic lights, allowing it to alert the drivers directly to overcome any sort of problems that may obstruct traffic flow. Furthermore, traffic lights can be managed by the CCTV system, by inputting the traffic load in a junction. Based on that, the system regulates the timing of traffic signals in real-time, taking in to account the variation in traffic demand [13].

Although the automated reaction approach might offer better traffic control, the process is very complicated and can be problematic; this is why it is always recommended to follow the system reactions closely and get them approved for safety. As argued by [16], it is better to have two cooperative monitoring procedures: the first is a spot-detection system generating vehicle count rate, speed, road occupancy and headway measures. The second is an extended-range road surveillance process to make decisions about suitable reactions and long term plans. An example of such a system is ARTEMIS, which stands for the Automatic Road Traffic Event Monitoring Information System. The mechanism of such system is that the extended-range camera will notice the vehicles starting to queue and the spot-detection system will confirm this by detecting the 
movement of traffic flow. If it is slower than the pre-defined speed threshold, then the system will send automatic alerts [11].

\section{CONSIDRATIONS AND LEGAL ISSUES}

CCTV leads to better traffic control. On the other hand, it is equally important to consider the disadvantages or possible threats that suggest that CCTV should not be used for traffic control. Indeed, there are distinct problems and issues associated with traffic control by CCTV:

CCTV systems are extremely costly to install and operate and may in fact not be the best form of traffic control in every case [8]. Therefore, the decision to install a CCTV traffic control system should be put in context. Sometimes, a transportation authority gets caught up in the rush to follow a popular trend or have unrealistic expectations about the system and push for something that is, in fact, neither necessary nor fits a particular traffic problem. Additionally, there are some indications that traffic in areas monitored by CCTV cameras are simply transferred to other areas with no CCTV cameras, as people would rather take unmonitored routs.

A big issue associated with CCTV systems is that they are offensive to privacy. Therefore, many countries have introduced a code of conduct for CCTV systems to insure that the systems are operated in a highly professional and private manner. The document sets guidelines for practice and procedures that should be followed, including: camera positions, sound facilities, video storage, video tapes as evidence, control rooms, etc. [11][17].

As stated in [18], a list of legal requirements must be met by a CCTV system. This includes:

- $\quad$ "CCTV should only be used in public places, i.e. in areas where persons do not have a reasonable expectation of privacy.

- $\quad$ Approval must be obtained from the owners of buildings and structures to which cameras will be attached.

- $\quad$ Personnel operating the system should be carefully screened and selected to ensure professionalism and trustworthiness since recorded material is sensitive and confidential.

- The recorded material must be treated as highly-confidential and be erased after a specified period of no longer than 30 days, unless needed for evidence. Material can, under no circumstances, be used for any purpose other than crime detection and evidence. Video tapes must be kept locked and there must be registers to control the storage of material.

- $\quad$ There should be sanctions attached to the transgression of guidelines.

- $\quad$ No promises or guarantees should be made to the public or any other role player about the system."

\section{CONCLUSION}

CCTV systems are increasingly used for road monitoring and traffic control systems. A typical CCTV system is composed of a camera system, reviewing displays and a central controller, beside possible additional accessories for lighting and movement controls. It is important to make the right decision regarding the number of cameras to be installed and their location, making sure to avoid possible road obstacles that might affects the quality of captured images.

The computer analysis process of CCTV data is important to reduce errors and cost in terms of time and money. This process, usually, involves pre-processing, to reduce irrelevant data and noise and then processing and analysis, to extract certain features. This step is sometimes 
followed by post-processing, to summarise long videos in particular. The final step is to react based on the analysed information. The reaction can be either manual or automatic.

Although CCTV systems offer many advantages in traffic control and management, ethical issues associated with them should be considered to make sure that people's privacy is not violated.

This review of the state-of-the-art in computer analysis of CCTV systems invites more detailed analysis and comparisons between available sound-based and image-based techniques and encourages hybrid approaches that combine the two.

\section{REFERENCES}

1. J. R. Agustina and G. Galdon Clavell, "The impact of CCTV on fundamental rights and crime prevention strategies: The case of the Catalan Control Commission of Video surveillance Devices," Computer Law \& Security Review, vol. 27, pp. 168-174, 4, 2011.

2. M. McCahill and C. Norris, "CCTV in London," Report Deliverable of UrbanEye Project, 2002.

3. M. McCahill and C. Norris, "CCTV in Britain," Center for Criminology and Criminal JusticeUniversity of Hull-United Kingdom, pp. 1-70, 2002

4. M. Courtney, "Public eyes get smart," Engineering \& Technology, vol. 6, pp. 38-41, 2011.

5. P. Kumar, S. Ranganath, H. Weimin, K. Sengupta, "A Framework for Real Time Behavior Interpretation from Traffic Video," Intelligent Transportation Systems, 2005, vol.6 .

6. L. Leontiadis, G. Marfia, D. Mack, G. Pau, C. Mascolo, M. Gerla, "On the Effectiveness of an Opportunistic Traffic Management System for Vehicular Networks," Intelligent Transportation Systems, Dec. 2011, vol. 12 (4).

7. G. Pratishtha, G. N. Purohit, "A Comparative Analysis of Traffic Control Technologies: CCTV versus RFTD,” National Conference on Data Management \& Security, 2011.

8. R. L. Bertini, C. Monsere, T. Yin, “

9. Benefits of Intelligent Transportation Systems Technologies in Urban Areas: A Literature Review," Portland State University, Center for Transportation Studies, Research Report, April 2005.

10. F. Conche and M. Tight, "Use of CCTV to determine road accident factors in urban areas," Accident Analysis \& Prevention, vol. 38, pp. 1197-1207, 11, 2006.

11. E. L. Piza, J. M. Caplan and L. W. Kennedy, "Is the Punishment More Certain? An Analysis of CCTV Detections and Enforcement," Justice Q., pp. 1-29.

12. G. Lorraine , F. Nacerodien, "An Assessment Of Closed Circuit Television Surveillance With Reference To The Benoni Project," Occasional Paper No. 1, Government of South Africa, August 2003.

13. Inter Pacific "All-seeing eye for CCTV surveillance," New Scientist, vol. 208, pp. 23, Nov 13-Nov 19, 2010.

14. P. Gupta and G. Purohit, "Computing optimum number of CCTV cameras for real-time traffic signal control system," in Parallel Distributed and Grid Computing (PDGC), 2012 2nd IEEE International Conference on, 2012, pp. 246-251.

15. B. Brown, CCTV in town centres: Three case studies. Crime detection and prevention series: Paper No. 68, London: Home Office Police Research Group, 1995.

16. N. Buch, M. Cracknell, J. Orwell and S. A. Velastin, "Vehicle localisation and classification in urban CCTV streams," Proc.16th ITS WC, pp. 1-8, 2009.

17. R. J. Blissett, C. Stennett and R. M. Day, "New techniques for digital CCTV processing in automatic traffic monitoring," in Vehicle Navigation and Information Systems Conference, 1993., Proceedings of the IEEE-IEE, 1993, pp. 137-140.

18. B. C. Welsh and D. P. Farrington, "Effects of Closed-Circuit Television on Crime," The ANNALS of the American Academy of Political and Social Science, vol. 587, pp. 110-135.

19. J. L. Koekemoer, Research report: Legal implications of the use of close circuit television (CCTV) surveillance in public Areas: National Busienss Initiative Against Crime. Legal Services. Pretoria: South African Police Service, 1996. 